\title{
An Asymptotic Derivation of Weakly Nonlinear Ray Theory
}

\author{
Phoolan Prasad \\ Department of Mathematics, Indian Institute of Science, Bangalore - 560012 \\ email : prasad@math.iisc.ernet.in
}

Key Words: Nonlinear wave propagation, ray theory, hyperbolic equations, caustic.

AMS Subject Clarification : 35L65, 35L67, 76L05, 76NI5, 65M99, 35Q25

\begin{abstract}
Using a method of expansion similar to Chapman - Enskog expansion, a new formal perturbation scheme based on high frequency approximation has been constructed. The scheme leads to an eikonal equation in which the leading order amplitude appears. The transport equation for the amplitude has been deduced with an error $O\left(\epsilon^{2}\right)$ where $\epsilon$ is the small parameter appearing in the high frequency approximation. On a length scale over which Choquet - Bruhat's theory is valid, this theory reduces to the former. The theory is valid on a much larger length scale and the leading order terms give the weakly nonlinear ray theory (WNLRT) of Prasad, which has been very successful in giving physically realistic results and also in showing that the caustic of a linear theory is resolved when nonlinear effects are included. The weak shock ray theory with infinite system of compatibility conditions also follows from this theory.
\end{abstract}

\section{Introduction}

A ray theory is a result of a mathematical method of finding an approximate value of the solution of a hyperbolic system of partial differential equations based on the high frequency approximation. The high frequency approximation implies that we can formally distinguish between the amplitude $w$ and a phase function $\phi(\mathbf{x}, t)$ whose level surfaces in $\mathbf{x}$-space (at a fixed $t$ ) define a one parameter family of wavefronts. In a ray theory we study the successive positions of a given wavefront and also attempt to calculate the amplitude distribution $w$ on it. The method, when applied to a linear system of equations, gives rise to linear rays whose equations decouple from the transport equation for the amplitude. Quite frequently, the linear rays starting from the points of a curved wavefront envelop a caustic surface on which the linear wavefront has cusp type of singularities where the assumptions of the ray theory break down. Thus the ray theory applied to a linear system is valid only over a distance which is small compared to the distance of

Proc. Indian Acad. Sci. (Math. Sci), Vol.110, No.4, November 2000, pp.1-17. 
an arête (where caustic begins to form) from the initial position of the wavefront. For a linear wavefront propagating in an uniform isotropic medium at rest, this distance $R$ of arête is equal to the minimum of the principal radii of curvature.

Experimental results (Sturtevant and Kulkarni, 1976) and theoretical investigations (Prasad 1993, and Prasad and Sangeeta, 1999) have shown that the amplitude of a wavefront, even when small amplitude assumption is made, has significant effect on rays and the wavefront geometry. Hence a nonlinear ray theory requires derivation of two equations, the first one being the eikonal equation

$$
Q\left(\nabla \phi, \phi_{t}, \mathbf{x}, t, w(\mathbf{x}, t)\right)=0, \quad \nabla=\left(\partial_{x_{1}}, \ldots, \partial_{x_{n}}\right)
$$

for the phase function $\phi$ such that the amplitude $w$ of the wavefront appears in the eikonal equation itself. The second equation is a transport equation for the amplitude along a nonlinear ray. The nonlinear rays are curves $\mathbf{x}=\mathbf{x}(t)$ obtained from the solution of the characteristic equations or Hamilton's canonical equations of (1.1). Though, ray theories for the propagation of a nonlinear wavefront were dealt by many (Keller (1954), Whitham (1956), Parker (1969, 1971); Choquet-Bruhat (1969) presented a systematic formal derivation of it for a general hyperbolic system of quasilinear partial differential equations. Hunter and his collaborators extended Choquet-Bruhat's theory to many important situations (Hunter, 1995). The Choquet-Bruhat's perturbation procedure leads to an eikonal equation which is independent of the amplitude $w$ and therefore, uses a transport equation for the amplitude along linear rays. Naturally the theory is valid over a distance over which a linear theory is valid i.e. on a length scale much smaller than $R$. Over a number of years, Prasad $(1975,1987,1993,1994)$ (see also Ravindran and Prasad, $1977,1985)$ has developed a nonlinear ray theory in which the eikonal equation depends also on the amplitude and the transport equation is along the nonlinear rays. The solution influences the wavefront geometry in such a way that the radii of curvature has no relevance as a length scale in the problem. Both experimental (Sturtevant and Kulkarni,1976) and theoretical results (Prasad 1993, and Prasad and Sangeeta, 1999) show that for a moderately weak nonlinear wave, the caustic does not appear in the solution. The wavefront geometry consists of almost plane segments joined by kinks across which the amplitude and the ray direction suffer jump. On each of these segments the amplitude of the wave varies slowly. Therefore, in a moderately weak nonlinear ray theory, the problem is not to find the solution in a caustic region because the caustic itself does not appear but to find the new geometry of a nonlinear wavefront and the solution on it. It turns out that except for immediate neighbourhoods of the kinks, the nonlinear ray theory is valid on a much larger length scale. The structure of the kinks can be studied on a smaller length scale by two-dimensional Burger's equations (or Zaboltskaya-Khokhlov or Z-K equation) which have been studied by Hunter and his coworkers (Hunter and Brio, 1992, 2000, and Hunter 1997) and Tabak and Rosales (1994).

The aim of this paper is to construct a formal perturbation scheme which leads to an eikonal equation in which the leading order amplitude $w$ appears and to derive a transport equation for $w$ along the corresponding nonlinear rays. We have been able to deduce the transport equation for $w$ with an error of the order of $\epsilon^{2}$. The method of expansion is similar to the Chapman-Enskog expansion, a discussion of which for a hyperbolic system is available in an article by Hunter (1995). A careful examination of the various terms in ray equations and the transport equation show that in practice only a few terms may be retained and this leads to the nonlinear ray theory of Prasad, which 
has been very successful in giving physically realistic results and also in showing that the caustic of a linear theory is resolved when nonlinear effect are included (Prasad and Sangeeta (1990) and Monica and Prasad (1989)). These two references contain extensive numerical results of the approximate equations derived in this paper. So does the paper of Kevlahan who shows that the shock ray theory derived in the end of the section 5 gives results which agrees well with experimental results, known expressions for approximate solutions and numerical solution of full Euler equations. A still better comparision with numerical solution of Euler equations is being worked out but this will take some time and will be reported later.

\section{An asymptotic derivation of WNLRT}

We consider a hyperbolic system of first order quasilinear partial differential equations,

$$
A(\mathbf{u}) \mathbf{u}_{t}+B^{(\alpha)}(\mathbf{u}) \mathbf{u}_{\mathbf{x}_{\alpha}}=0 \quad \alpha=1,2, \ldots m
$$

Here, $\mathbf{x} \in R^{m}$ are the space variables, $\mathbf{u}(\mathbf{x}, t) \in R^{n}$ are the dependent variables, and $A$ and $B^{(\alpha)}(\mathbf{u})$, are smooth $n \times n$ matrix-valued functions of $\mathbf{u}$. We use the summation convention over repeated indices. We only consider smooth solutions, so it is not necessary to write the system in conservation form.

We look for a generalized asymptotic expansion of solutions of (2.1) of the following form:

$$
\begin{gathered}
\mathbf{u}(\mathbf{x}, t, \epsilon)=\epsilon \mathbf{v}\left(\mathbf{x}, t, \frac{\phi(\mathbf{x}, t, \epsilon)}{\epsilon}, \epsilon\right) \\
\mathbf{v}(\mathbf{x}, t, \theta, \epsilon)=\mathbf{v}_{0}(\mathbf{x}, t, \theta, \epsilon)+\epsilon \mathbf{v}^{\prime}(\mathbf{x}, t, \theta, \epsilon), \quad \theta=\phi / \epsilon
\end{gathered}
$$

Here $\epsilon$ is a small parameter, so this ansatz represents a small amplitude high frequency solution. The function $\phi(\mathbf{x}, t, \epsilon) \in R$ and the functions $\mathbf{v}_{0}(\mathbf{x}, t, \theta, \epsilon)$ and $\mathbf{v}^{\prime}(\mathbf{x}, t, \theta, \epsilon)$ will be chosen so that (2.2) gives an asymptotic solution of (2.1) as $\epsilon \rightarrow 0$. In particular, $\phi$ is the phase function associated with the leading order solution $\mathbf{u}=\epsilon \mathbf{v}_{0}$. When carrying out the expansion, we assume that the derivatives, $\phi_{x_{\alpha}}$, are of order one with respect to $\epsilon$.

This method of expansion is similar to the Chapman-Enskog expansion . For other work in nonlinear hyperbolic waves using Chapman-Enskog expansions see Hunter (1995). The leading order solution $\mathbf{v}_{0}$ and the correction $\mathbf{v}^{\prime}$ depend on $\epsilon$ explicitly. It is therefore not necessary to include any higher order terms in the expansion (2.3), since they can be absorbed into $\mathbf{v}^{\prime}$. As a result of this explicit $\epsilon$ dependence, the solution $\mathbf{v}$ can be decomposed into a leading order approximation, $\mathbf{v}_{0}$ and a perturbation $\epsilon \mathbf{v}^{\prime}$ in different ways, since terms in $\mathbf{v}^{\prime}$ can be absorbed into $\mathbf{v}_{0}$. One way to specify the decomposition uniquely is to require that

$$
\mathbf{l}_{0} \cdot \mathbf{v}^{\prime}=0
$$

where the left null vector $\mathbf{l}_{\mathbf{0}}$ is defined below. However, other choices are possible. For example in gas dynamics we could require that $\mathbf{v}^{\prime}$ contains no pressure perturbations.

We now derive the asymptotic equations. We will obtain an asymptotic solution which satisfies (2.1) upto terms of the order $\epsilon^{3}$. Higher order approximations can be derived in 
a similar way, although the resulting equations rapidly become very complicated. Use of $(2.2)$ in $(2.1)$ gives

$$
\left\{\phi_{t} A(\epsilon \mathbf{V})+\phi_{\mathbf{x}_{\alpha}} B^{(\alpha)}(\epsilon \mathbf{v})\right\} \mathbf{v}_{\theta}+\epsilon\left\{A(\epsilon \mathbf{v}) \mathbf{v}_{t}+B^{(\alpha)}(\epsilon \mathbf{V}) \mathbf{v}_{\mathbf{x}_{\alpha}}\right\}=0
$$

Here, $\mathbf{v}_{\theta}$ is the partial derivative of $\mathbf{v}$ at fixed $\mathbf{x}, t$ and $\mathbf{v}_{t}, \mathbf{v}_{\mathbf{x}_{\alpha}}$ are the partial derivatives at a fixed $\theta$.

We note that if $\mathbf{v}(\mathbf{x}, t, \theta, \epsilon)$ satisfies (2.5) when $\theta=\epsilon^{-1} \phi(\mathbf{x}, t, \epsilon)$ (rather than for all $\theta$, as is usually assumed in the method of multiple scales), then (2.2) gives a solution of the original equation (2.1). We are therefore free to regard any coefficient in (2.5) which do not contain derivatives as functions of $\mathbf{x}, t$ with $\theta$ evaluated at $\frac{\phi(\mathbf{x}, t, \epsilon)}{\epsilon}$. Using (2.3) in (2.5) and Taylor expanding the coefficient matrices, we obtain

$$
\begin{aligned}
& \left\{\phi_{t} A\left(\epsilon \mathbf{v}_{0}\right)+\phi_{x_{\alpha}} B^{(\alpha)}\left(\epsilon \mathbf{v}_{0}\right)\right\} \mathbf{v}_{0 \theta} \\
+ & \epsilon\left\{\left[\phi_{t} A\left(\epsilon \mathbf{v}_{0}\right)+\phi_{x_{\alpha}} B^{(\alpha)}\left(\epsilon \mathbf{v}_{0}\right)\right] \mathbf{v}_{\theta}^{\prime}+A\left(\epsilon \mathbf{v}_{0}\right) \mathbf{v}_{0 t}+B^{(\alpha)}\left(\epsilon \mathbf{v}_{0}\right) \mathbf{v}_{0 x_{\alpha}}\right\} \\
+ & \epsilon^{2}\left\{\left[\phi_{t}\left(\nabla_{\mathbf{u}} A\right)\left(\epsilon \mathbf{v}_{0}\right) \cdot \mathbf{v}^{\prime}+\phi_{x_{\alpha}}\left(\nabla_{\mathbf{u}} B^{(\alpha)}\right)\left(\epsilon \mathbf{v}_{0}\right) \cdot \mathbf{v}^{\prime}\right] \mathbf{v}_{0 \theta}+A\left(\epsilon \mathbf{v}_{0}\right) \mathbf{v}_{t}^{\prime}+B^{(\alpha)}\left(\epsilon \mathbf{v}_{0}\right) \mathbf{v}_{x_{\alpha}}^{\prime}\right\} \\
= & O\left(\epsilon^{3}\right)
\end{aligned}
$$

As we remarked above $\mathbf{v}(\mathbf{x}, t, \theta, \epsilon)$ is only required to satisfy this equation when $\theta=\frac{\phi}{\epsilon}$. We can therefore evaluate all the coefficients at this value of $\theta$ to obtain the equation

$$
\begin{gathered}
{\left[\phi_{t} A_{0}+\phi_{x_{\alpha}} B_{0}^{(\alpha)}\right] \mathbf{v}_{0 \theta}+\epsilon\left\{\left(\phi_{t} A_{0}+\phi_{x_{\alpha}} B_{0}^{(\alpha)}\right) \mathbf{v}_{\theta}^{\prime}+A_{0} \mathbf{v}_{0 t}+B_{0}^{(\alpha)} \mathbf{v}_{0 x_{\alpha}}\right\}} \\
+\epsilon^{2}\left[\left\{\phi_{t}\left(\nabla_{\mathbf{u}} A\right)_{0} \cdot \mathbf{v}^{\prime}+\phi_{x_{\alpha}}\left(\nabla_{\mathbf{u}} B^{(\alpha)}\right)_{0} \cdot \mathbf{v}^{\prime}\right\} \mathbf{v}_{0 \theta}+A_{0} \mathbf{v}_{t}^{\prime}+B_{0}^{(\alpha)} \mathbf{v}_{x_{\alpha}}^{\prime}\right]=O\left(\epsilon^{3}\right)
\end{gathered}
$$

where the subscript 0 indicates that the coefficients are evaluated at $\mathbf{u}=\epsilon \mathbf{v}_{0}(\mathbf{x}, t$, $\left.\epsilon^{-1} \phi(t, \mathbf{x}, \epsilon), \epsilon\right)$ so that they are functions of $\mathbf{x}, t$ and $\epsilon$. For example,

$$
B_{0}^{(\alpha)}(\mathbf{x}, t, \epsilon)=B^{(\alpha)}\left(\epsilon \mathbf{v}_{0}\left(\mathbf{x}, t, \epsilon^{-1} \phi(\mathbf{x}, t, \epsilon), \epsilon\right)\right)
$$

The three terms in (2.7) are not completely separated as coefficients of the powers of $\epsilon^{0}, \epsilon$ and $\epsilon^{2}$ are also dependent on $\epsilon$. The first term, which is of order 1, vanishes up to this order and, therefore, we impose that it is exactly zero i.e.

$$
\left\{\phi_{t} A_{0}+\phi_{x_{\alpha}} B_{0}^{(\alpha)}\right\} \mathbf{v}_{0 \theta}=0
$$

When we choose the leading term $\mathbf{v}_{0}$ in the high frequency asymptotic limit $\epsilon \rightarrow 0$ to satisfy this equation, the first term in (2.7) vanishes and we get a relation

$$
\begin{aligned}
& \left\{\phi_{t} A_{0}+\phi_{x_{\alpha}} B_{0}^{(\alpha)}\right\} \mathbf{v}_{\theta}^{\prime}+A_{0} \mathbf{v}_{0 t}+B_{0}^{(\alpha)} \mathbf{v}_{0 x_{\alpha}} \\
+ & \epsilon\left\{\left[\phi_{t}\left(\nabla_{\mathbf{u}} A\right)_{0} \cdot \mathbf{v}^{\prime}+\phi_{x_{\alpha}}\left(\nabla_{\mathbf{u}} B^{(\alpha)}\right)_{0} \cdot \mathbf{v}^{\prime}\right] \mathbf{v}_{0 \theta}+A_{0} \mathbf{v}_{t}^{\prime}+B_{0}^{(\alpha)} \mathbf{v}_{x_{\alpha}}^{\prime}\right\}=O\left(\epsilon^{2}\right)
\end{aligned}
$$

between $\mathbf{v}_{0}$ and $\mathbf{v}^{\prime}$ with error of the order $\epsilon^{2}$. To obtain a nontrivial solution for $\mathbf{v}_{0}$, we then require that $\phi$ satisfies the eikonal equation

$$
\operatorname{det}\left[\phi_{t} A_{0}(\mathbf{x}, t, \epsilon)+\phi_{x_{\alpha}}(\mathbf{x}, t, \epsilon) B_{0}^{(\alpha)}(\mathbf{x}, t, \epsilon)\right]=0
$$

We note that this eikonal equation is associated with the function $\mathbf{u}=\epsilon \mathbf{v}_{0}\left(\mathbf{x}, t, \epsilon^{-1}\right.$ $\phi(\mathbf{x}, t, \epsilon), \epsilon)$ and thus we are able to incorporate leading order wave amplitude correction 
in the eikonal equation itself. We denote left and right null vectors associated with the phase $\phi(t, \mathbf{x}, \epsilon)$ and the perturbed state $u=\epsilon \mathbf{v}_{0}$ by $\mathbf{l}_{0}(\mathbf{x}, t, \epsilon)$ and $\mathbf{r}_{0}(\mathbf{x}, t, \epsilon)$, respectively, i.e $\mathbf{l}_{0}$ and $\mathbf{r}_{0}$ satisfy

$$
\mathbf{l}_{0} \cdot\left(\phi_{t} A_{0}+\phi_{x_{\alpha}} B_{0}^{(\alpha)}\right)=0
$$

and

$$
\left(\phi_{t} A_{0}+\phi_{x_{\alpha}} B_{0}^{(\alpha)}\right) \mathbf{r}_{0}=0
$$

Here

$$
\begin{aligned}
& \mathbf{l}_{0}(\mathbf{x}, t, \epsilon)=\mathbf{l}\left(\mathbf{n}(\mathbf{x}, t, \epsilon), \epsilon \mathbf{v}_{0}\right) \\
& \mathbf{r}_{0}(\mathbf{x}, t, \epsilon)=\mathbf{r}\left(\mathbf{n}(\mathbf{x}, t, \epsilon), \epsilon \mathbf{v}_{0}\right)
\end{aligned}
$$

where

$$
\mathbf{n}(\mathbf{x}, t, \epsilon)=\frac{\nabla \phi}{|\nabla \phi|}, \quad \nabla \phi=\left(\phi_{x_{1}}, \phi_{x_{2}}, \ldots, \phi_{x_{m}}\right)
$$

Also we normalize $\mathbf{l}_{0}$ so that

$$
\mathbf{l}_{0} A_{0} \mathbf{r}_{0}=1
$$

A solution of (2.9) is given by

$$
\mathbf{v}_{0}(\mathbf{x}, t, \theta, \epsilon)=w(\mathbf{x}, t, \theta, \epsilon) \mathbf{r}_{0}(\mathbf{x}, t, \epsilon)
$$

where $w$ is an arbitrary scalar valued amplitude function. Taking the scalar product of (2.10) with the left null vector $\mathbf{l}_{0}$ we obtain

$$
\begin{aligned}
& \mathbf{l}_{0}\left(A_{0} \mathbf{v}_{0 t}+B_{0}^{(\alpha)} \mathbf{v}_{0 x_{\alpha}}\right) \\
+ & \epsilon \mathbf{l}_{0}\left\{\left[\phi_{t}\left(\nabla_{\mathbf{u}} A\right)_{0} \cdot \mathbf{v}^{\prime}+\phi_{x_{\alpha}}\left(\nabla_{\mathbf{u}} B^{(\alpha)}\right)_{0} \cdot \mathbf{v}^{\prime}\right] \mathbf{v}_{0 \theta}+A_{0} \mathbf{v}_{t}^{\prime}+B_{0}^{(\alpha)} \mathbf{v}_{x_{\alpha}}^{\prime}\right\} \\
= & O\left(\epsilon^{2}\right)
\end{aligned}
$$

To eliminate $\mathbf{v}^{\prime}$ from this equation, we solve (2.10) iteratively for $\mathbf{v}^{\prime}$ in terms of $\mathbf{v}_{0}$. In order that the eliminant has an error of order $\epsilon^{2}$ consistent with (2.19), we note that we need to solve $\mathbf{v}^{\prime}$ with error of order $\epsilon$ i.e we consider only the leading order terms in $(2.10)$

$$
\left\{\phi_{t} A_{0}+\phi_{x_{\alpha}} B_{0}^{(\alpha)}\right\} \mathbf{v}_{\theta}^{\prime}+A_{0} \mathbf{v}_{0 t}+B_{0}^{(\alpha)} \mathbf{v}_{0 x_{\alpha}}=O(\epsilon)
$$

We use (2.18) in (2.20). A solution of the resulting equation for $\mathbf{v}^{\prime}$ is then

$$
\mathbf{v}^{\prime}(\mathbf{x}, t, \theta, \epsilon)=b_{t}(\mathbf{x}, t, \theta, \epsilon) \mathbf{s}_{0}^{\prime}+b_{x_{\beta}}(\mathbf{x}, t, \theta, \epsilon) \mathbf{s}_{0}^{(\beta)}+b(\mathbf{x}, t, \theta, \epsilon) \mathbf{s}_{0}+O(\epsilon)
$$

where $b$ is the scalar amplitude such that

$$
b_{\theta}=w
$$

and the vectors $\mathbf{s}_{0}(\mathbf{x}, t, \epsilon), \mathbf{s}_{0}^{\prime}(t, \mathbf{x}, \epsilon)$ and $\mathbf{s}_{0}^{(\beta)}(\mathbf{x}, t, \epsilon)$ satisfy

$$
\begin{gathered}
\left(\phi_{t} A_{0}+\phi_{x_{\alpha}} B_{0}^{(\alpha)}\right) \mathbf{s}_{0}=-\left(A_{0} \mathbf{r}_{0 t}+B_{0}^{(\alpha)} \mathbf{r}_{0 x_{\alpha}}\right)+\left(\mathbf{l}_{0}\left(A_{0} \mathbf{r}_{0 t}+B_{0}^{(\alpha)} \mathbf{r}_{0 x_{\alpha}}\right)\right) A_{0} \mathbf{r}_{0} \\
\left(\phi_{t} A_{0}+\phi_{x_{\alpha}} B_{0}^{(\alpha)}\right) \mathbf{s}_{0}^{\prime}=-\left(A_{0} \mathbf{r}_{0}\right)+\left(\mathbf{l}_{0} A_{0} \mathbf{r}_{0}\right) A_{0} \mathbf{r}_{0} \\
\left(\phi_{t} A_{0}+\phi_{x_{\alpha}} B_{0}^{(\alpha)}\right) \mathbf{s}_{0}^{\beta}=-\left(B_{0}^{(\beta)} \mathbf{r}_{0}\right)+\left(\mathbf{l}_{0} B_{0}^{(\beta)} \mathbf{r}_{0}\right) A_{0} \mathbf{r}_{0}
\end{gathered}
$$


These equations do not have a unique solution. This is because there is some arbitrariness in how $\mathbf{v}$ is decomposed into $\mathbf{v}_{0}$ and $\mathbf{v}^{\prime}$. But if we impose the condition (2.4) on $\mathbf{v}^{\prime}$, then we choose the unique solutions of (2.23) - (2.24) such that

$$
\mathbf{l}_{0} \mathbf{s}_{0}=\mathbf{l}_{0} \mathbf{s}_{0}^{\prime}=\mathbf{l}_{0} \mathbf{s}_{0}{ }^{\beta}=0
$$

Finally, use of (2.18) and (2.21) in (2.19) gives the following transport equation for $w$,

$$
w_{t}+\chi_{\alpha_{0}} w_{x_{\alpha}}-\Omega w+\epsilon\left[\left(\Gamma^{t} b_{t}+\Gamma^{\alpha} b_{x_{\alpha}}+\Gamma b\right) w_{\theta}+W b_{t}+V^{\alpha} b_{x_{\alpha}}+D^{\alpha \beta}+E b\right]=O\left(\epsilon^{2}\right)
$$

Note that $D^{\alpha \beta}$ contains linear terms in the second order derivatives of $b$ as seen below. The coefficients are functions of $(\mathbf{x}, t, \epsilon)$ given by

$$
\left.\begin{array}{ccc}
\chi_{\alpha_{0}}= & \mathbf{l}_{0} B_{0}^{(\alpha)} \mathbf{r}_{0} \\
\Omega & = & \\
\Gamma & = & \mathbf{l}_{0}\left(\phi_{t}\left(\nabla_{\mathbf{u}} A\right)_{0}+\phi_{x_{\alpha}}\left(\nabla_{\mathbf{u}} B^{(\alpha)}\right)_{0}\right) \cdot \mathbf{s}_{0} \mathbf{r}_{0} \\
\Gamma^{t}= & \mathbf{l}_{0}\left(\phi_{t}\left(\nabla_{\mathbf{u}} A\right)_{0}+\phi_{x_{\beta}}\left(\nabla_{\mathbf{u}} B^{(\beta)}\right)_{0}\right) \cdot \mathbf{s}_{0}^{\prime} \mathbf{r}_{0} \\
\Gamma^{\alpha}= & \mathbf{l}_{0}\left(\phi_{t}\left(\nabla_{\mathbf{u}} A\right)_{0}+\phi_{x_{\beta}}\left(\nabla_{\mathbf{u}} B^{(\beta)}\right)_{0}\right) \cdot \mathbf{s}_{0}^{(\alpha)} \mathbf{r}_{0} \\
W & = & \mathbf{l}_{0}\left(A_{0} \mathbf{s}_{0}+A_{0} \mathbf{s}_{0 t}^{\prime}+B_{0}^{(\beta)} \mathbf{s}_{0 x_{\beta}}^{\prime}\right) \\
V^{\alpha}= & \mathbf{l}_{0}\left(B_{0}^{(\alpha)} \mathbf{s}_{0}+A_{0} \mathbf{s}_{0 t}^{(\alpha)}+B_{0}^{(\beta)} \mathbf{s}_{0 x_{\beta}}^{(\alpha)}\right) \\
D^{\alpha \beta}= & \mathbf{l}_{0}\left\{A_{0} \mathbf{s}_{0}^{\prime} b_{t t}+A_{0} \mathbf{s}_{0}^{(\beta)} b_{x_{\beta} t}+B_{0}^{(\alpha)} \mathbf{s}_{0}^{\prime} b_{t x_{\alpha}}+B_{0}^{(\alpha)} \mathbf{s}_{0}^{(\beta)} b_{x_{\alpha} x_{\beta}}\right.
\end{array}\right\}
$$

\section{Ray formulation of the Asymptotic equations}

The eikonal equation (2.9) can be equivalently written in the form

$$
Q \equiv \phi_{t}\left(\mathbf{l}_{0} A_{0} \mathbf{r}_{0}\right)+\phi_{x_{\alpha}}\left(\mathbf{l}_{0} B_{0}^{(\alpha)} \mathbf{r}_{0}\right)=0, \quad \alpha=1, \ldots, m
$$

From the characteristic equations of (3.1) we obtain

$$
\begin{gathered}
\frac{d x_{\alpha}}{d s}=\frac{\partial Q}{\partial \phi_{x^{\alpha}}}=\mathbf{l}_{0} B_{0}^{(\alpha)} \mathbf{r}_{0}=\chi_{\alpha_{0}} \\
\frac{d t}{d s}=\frac{\partial Q}{\partial \phi_{t}}=\mathbf{l}_{0} A_{0} \mathbf{r}_{0}=1 \\
\frac{d \phi_{x_{\alpha}}}{d s}=-\frac{\partial Q}{\partial x^{\alpha}}=\phi_{t}\left(\mathbf{l}_{0} A_{0 x_{\alpha}} \mathbf{r}_{0}\right)+\phi_{x_{\gamma}}\left(\mathbf{l}_{0} B_{0 x^{\alpha}}^{(\gamma)} \mathbf{r}_{0}\right), \quad \gamma=1, \ldots, m
\end{gathered}
$$


Now for a fixed $t, \phi(\mathbf{x}, t, \epsilon)=0$ represents a wavefront in $\mathbf{x}-$ space with unit normal

$$
\mathbf{n}=\frac{\nabla \phi}{|\nabla \phi|}, \quad \nabla=\left(\partial_{x_{1}}, \ldots, \partial_{x_{m}}\right)
$$

The differential equation for $\mathbf{n}$ is

$$
\frac{d n_{\alpha}}{d s}=-n_{\beta} \mathbf{l}_{0}\left(-c_{0} \frac{\partial A_{0}}{\partial \eta_{\beta}^{\alpha}}+n_{\gamma} \frac{\partial B_{0}^{\gamma}}{\partial \eta_{\beta}^{\alpha}}\right) \mathbf{r}_{0} \equiv \Psi_{\alpha_{0}}, \quad \text { say }
$$

where

$$
\frac{\partial}{\partial \eta_{\beta}^{\alpha}}=n_{\beta} \frac{\partial}{\partial x_{\alpha}}-n_{\alpha} \frac{\partial}{\partial x^{\beta}} \quad \beta=1,2, \ldots, m
$$

and

$$
c_{0}=\frac{\phi_{t}}{|\nabla \phi|}
$$

The operator

$$
\frac{d}{d s}=\frac{\partial}{\partial t}+\chi_{\alpha_{0}} \frac{\partial}{\partial x_{\alpha}}
$$

appearing on the left hand side of (3.2)-(3.4) and (3.5), and $\frac{\partial}{\partial \eta_{\beta}^{\alpha}}$ defined above are in direction tangential to a characteristic surface $\phi(\mathbf{x}, t)=$ constant in $(\mathbf{x}, t)$ space. In addition the derivatives $\frac{\partial}{\partial \eta_{\beta}^{\alpha}}$ are tangential to a wavefront $\phi(\mathbf{x}, t)=$ constant with $t=$ constant in $\left(x_{1}, \ldots, x_{m}\right)$ - space. Because of the choice $(2.17), \frac{d}{d s}$ represents time rate of change along a ray and may be denoted by the symbol $\frac{d}{d t}$. The transport equation (2.26) can then be written as

$$
\frac{d w}{d s}=\Omega w-\epsilon\left[\left(\Gamma^{t} b_{t}+\Gamma^{\alpha} b_{x_{\alpha}}+\Gamma b\right) w_{\theta}+W b_{t}+V^{\alpha} b_{x_{\alpha}}+D^{\alpha \beta}+E b\right]+O\left(\epsilon^{2}\right)
$$

The equations (3.2), (3.5) and (3.9) form a complete set of equations of the nonlinear ray theory with error $O\left(\epsilon^{2}\right)$. The amplitude $\mathbf{u}=\epsilon \mathbf{v}_{\mathbf{0}}=\epsilon w \mathbf{r}_{0}$ upto first order in $\epsilon$ appears in the bicharacteristic velocity $\chi_{\alpha_{0}}(\mathbf{x}, t, \epsilon)$ and the rate of turning $\Psi_{\alpha_{0}}$ of the rays, given in a complicated way.

The interesting and important point of this weakly nonlinear ray theory (WNLRT) is that the transport equation (3.9) for $w$ along nonlinear rays is coupled to the ray equations (3.2) and (3.5), which correspond to the leading order wave a amplitude $w$. Earlier transport equation, derived by Gubkin (1958) for gasdynamics equation and by Prasad (1975) for a general hyperbolic system, were along the exact nonlinear rays corresponding to the exact solution $\mathbf{u}$ in the form (2.2). Gubkin and Prasad derived the transport equation on geometrical consideration by approximating the system (2.1) in the neighbourhood of the exact characteristic surface in space-time. Proper interpretation of transport equation along the nonlinear rays corresponding to leading order amplitude $w$ has lead to physically realistic solutions (Ravindran and Prasad (1985), Prasad (1987, 1993) and Prasad and Sangeeta (1999)). 
To make these equations more tractable, we approximate $\mathbf{l}_{0}$ and $\mathbf{r}_{0}$ defined by $(2.12)$ and (2.13) as follows. We now define $\overline{\mathbf{l}}$ and $\overline{\mathbf{r}}$ as

$$
\overline{\mathbf{l}}=\mathbf{l}_{0}(\mathbf{n}, 0) \quad \overline{\mathbf{r}}=\mathbf{r}_{0}(\mathbf{n}, 0)
$$

Then

$$
\begin{aligned}
\mathbf{l}_{0} & =\overline{\mathbf{l}}+\epsilon\left\{\left(\nabla_{\mathbf{u}} \mathbf{l}\right)_{0} \cdot \mathbf{v}_{0}\right\}+O\left(\epsilon^{2}\right) \\
& =\overline{\mathbf{l}}+\epsilon\left\{\left(\nabla_{\mathbf{u}} \mathbf{l}\right)_{0} \cdot \mathbf{r}_{0}\right\} w+O\left(\epsilon^{2}\right)
\end{aligned}
$$

where $\left(\nabla_{\mathbf{u}} \mathbf{l}\right)_{0}$ is the value of $\left(\nabla_{\mathbf{u}} \mathbf{l}\right)$ evaluated at $\mathbf{u}=0$ keeping $\mathbf{n}$ fixed and is a notation different from that introduced by equation (2.8) for the use of the subscript 0. Similarly

$$
\mathbf{r}_{0}=\overline{\mathbf{r}}+\epsilon\left\{\left(\nabla_{\mathbf{u}} \mathbf{r}\right)_{0} \cdot \mathbf{r}_{0}\right\} w+O\left(\epsilon^{2}\right)
$$

The vectors $\overline{\mathbf{l}}$ and $\overline{\mathbf{r}}$ still depend on the leading order term $\mathbf{v}_{0}$ in the solution and the nonlinear phase $\phi$, through $\mathbf{n}$. Also if

$$
A_{*}=A(\mathbf{u}=0) \text { and } B_{*}^{(\alpha)}=B^{(\alpha)}(\mathbf{u}=0) \text { are constant matrices }
$$

then we have

$$
A_{0}=A_{*}+\epsilon\left(\nabla_{u} A\right)_{*} \cdot \mathbf{r}_{0} w+O\left(\epsilon^{2}\right)
$$

and

$$
B_{0}^{(\alpha)}=B_{*}^{(\alpha)}+\epsilon\left(\nabla_{u} B^{(\alpha)}\right)_{*} \cdot \mathbf{r}_{0} w+O\left(\epsilon^{2}\right)
$$

where $\left(\nabla_{\mathbf{u}} B^{(\alpha)}\right)_{*}$ is the value of $\left(\nabla_{\mathbf{u}} B^{(\alpha)}\right)$ evaluated at $\mathbf{u}=0$. The important point in simplifying the equations now is to realise that a nonlinear wavefront given by the phase function $\phi(\mathbf{x}, t, \epsilon)$ may differ significantly from the corresponding linear wavefront given by the linear phase function $\phi^{*}(\mathbf{x}, t)$. This can be seen from the large number of results we have presented in the earlier papers including that by Prasad and Sangeeta (1999). The partial derivatives $\phi_{x_{\alpha}}$ of the nonlinear phase and $\phi_{x_{\alpha}}^{*}$ of the linear phase (i.e the unit normal $\mathbf{n}$ of a nonlinear wavefront and $\mathbf{n}_{*}$ of the corresponding linear wavefront) also differ significantly. One may think that the nonlinear ray theory which is being considered here may be valid only on the length scale over which the linear theory or Choquet-Bruhat's nonlinear theory are valid. But this is not so. In the derivation of this theory we have made no reference to the length scales associated with the linear theory. The numerical results of Prasad and Sangeeta (1999) show that this theory is valid even in a caustic region where the normal $\mathbf{n}$ of a nonlinear wavefront and $\mathbf{n}_{*}$ of the corresponding linear wavefront differ very much. Infact, the theory is valid on much larger length scale than the radii of curvature of the initial wavefront. Therefore, while trying to make further approximation in some of the terms in (3.2), (3.3), (3.5) and (3.9) we keep $\mathbf{n}$ and the operators $\frac{\partial}{\partial \eta_{\beta}^{\alpha}}$ (tangential derivatives on the nonlinear wavefront) unchanged and use Taylor's expansion with respect to $\epsilon \mathbf{v}_{\mathbf{0}}$ at $\mathbf{0}$. Following this we can approximate some of the terms as follows

$$
\begin{aligned}
\mathbf{l}_{0} B_{0}^{(\alpha)} \mathbf{r}_{0} & =\overline{\mathbf{l}} B_{*}^{(\alpha)} \overline{\mathbf{r}}+\epsilon\left[\left(\left(\nabla_{\mathbf{u}} \mathbf{l}\right)_{0} \cdot \overline{\mathbf{r}}\right) B_{*}^{(\alpha)} \overline{\mathbf{r}}+\overline{\mathbf{l}}\left(\left(\nabla_{\mathbf{u}} B^{(\alpha)}\right)_{*} \cdot \overline{\mathbf{r}}\right) \overline{\mathbf{r}}+\right. \\
& \left.+\overline{\mathbf{l}} B_{*}^{(\alpha)}\left(\nabla_{\mathbf{u}} \mathbf{r}\right)_{0} \cdot \overline{\mathbf{r}}\right] w+O\left(\epsilon^{2}\right)
\end{aligned}
$$




$$
\begin{aligned}
\mathbf{l}_{0} A_{0} \mathbf{r}_{0 t} & =\overline{\mathbf{l}} A_{*} \overline{\mathbf{r}}_{t}+\epsilon\left[\left(\left(\nabla_{\mathbf{u}} \mathbf{l}\right)_{0} \cdot \overline{\mathbf{r}}\right) A_{*} \overline{\mathbf{r}}_{t}+\overline{\mathbf{l}}\left(\left(\nabla_{\mathbf{u}} A\right)_{*} \cdot \overline{\mathbf{r}}\right) \overline{\mathbf{r}}_{t}+\overline{\mathbf{l}} A_{*}\left(\nabla_{\mathbf{u}} \mathbf{r}\right)_{0 t} \cdot \overline{\mathbf{r}}\right. \\
& \left.+\overline{\mathbf{l}} A_{*}\left(\nabla_{\mathbf{u}} \mathbf{r}\right)_{0} \cdot \overline{\mathbf{r}}_{t}\right] w+\epsilon \overline{\mathbf{l}} A_{*}\left(\left(\nabla_{\mathbf{u}} \mathbf{r}\right)_{0} \cdot \overline{\mathbf{r}}\right) w_{t}+O\left(\epsilon^{2}\right)
\end{aligned}
$$

and

$$
\begin{aligned}
\mathbf{l}_{0} B_{0} \mathbf{r}_{0_{x_{\alpha}}} & =\overline{\mathbf{l}} B_{*}^{(\alpha)} \overline{\mathbf{r}}+\epsilon\left[\left(\left(\nabla_{\mathbf{u}} \mathbf{l}\right)_{0} \cdot \overline{\mathbf{r}}\right) B_{*}^{(\alpha)} \overline{\mathbf{r}}_{x_{\alpha}}+\overline{\mathbf{l}}\left(\left(\nabla_{\mathbf{u}} B^{(\alpha)}\right)_{*} \cdot \overline{\mathbf{r}}\right) \overline{\mathbf{r}}_{x_{\alpha}}+\overline{\mathbf{l}} A_{*}\left(\nabla_{\mathbf{u}} \overline{\mathbf{r}}\right)_{0 x_{\alpha}} \cdot \overline{\mathbf{r}}\right. \\
& \left.+\overline{\mathbf{l}} B_{*}^{(\alpha)}\left(\nabla_{\mathbf{u}} \mathbf{r}\right)_{0} \cdot \overline{\mathbf{r}}_{x_{\alpha}}\right] w+\epsilon \overline{\mathbf{l}} B_{*}^{(\alpha)}\left(\left(\nabla_{\mathbf{u}} \mathbf{r}\right)_{0} \cdot \overline{\mathbf{r}}\right) w_{x_{\alpha}}+O\left(\epsilon^{2}\right)
\end{aligned}
$$

Therefore

$$
\mathbf{l}_{0} A_{0} \mathbf{r}_{0 t}+\mathbf{l}_{0} B_{0}^{(\alpha)} \mathbf{r}_{0 x_{\alpha}}=\overline{\mathbf{l}} A_{*} \overline{\mathbf{r}}_{t}+\overline{\mathbf{l}} B_{*}^{(\alpha)} \overline{\mathbf{r}}_{x_{\alpha}}+O(\epsilon)=-\bar{\Omega}+O(\epsilon)
$$

where

$$
\bar{\Omega}=-\left(\overline{\mathbf{l}} A_{*} \overline{\mathbf{r}}_{t}+\overline{\mathbf{l}} B_{*}^{(\alpha)} \overline{\mathbf{r}}_{x_{\alpha}}\right)
$$

Substituting (3.15) to (3.19) in (3.2), (3.5) and (3.9) and retaining terms only upto order $\epsilon$ we get the full set of equations of WNLRT (note $d / d s=d / d t$ )

$$
\begin{aligned}
\frac{d x_{\alpha}}{d t}=\overline{\mathbf{l}} B_{*}^{\alpha} \overline{\mathbf{r}}+\epsilon\left[\left(\left(\nabla_{\mathbf{u}} \mathbf{l}\right)_{0} \cdot \overline{\mathbf{r}}\right) B_{*}^{(\alpha)} \overline{\mathbf{r}}+\overline{\mathbf{l}}\left(\left(\nabla_{\mathbf{u}} B^{(\alpha)}\right)_{*} \cdot \overline{\mathbf{r}}\right) \overline{\mathbf{r}}+\right. \\
\left.+\overline{\mathbf{l}} B_{*}^{(\alpha)}\left(\nabla_{\mathbf{u}} \mathbf{r}\right)_{0} \cdot \overline{\mathbf{r}}\right] w+O\left(\epsilon^{2}\right) \\
\frac{d n_{0}^{\alpha}}{d t}=-\epsilon \mathbf{n}^{\beta} \overline{\mathbf{l}}\left[\left\{-\bar{c}\left(\nabla_{\mathbf{u}} A\right)_{*} \cdot \frac{\partial \overline{\mathbf{r}}}{\partial \eta_{\beta}^{\alpha}}+n_{\gamma}\left(\nabla_{\mathbf{u}} B^{\gamma}\right)_{*} \cdot \frac{\partial \overline{\mathbf{r}}}{\partial \eta_{\beta}^{\alpha}}\right\} w\right. \\
\left.+\left\{-\bar{c}\left(\nabla_{\mathbf{u}} A\right)_{*} \cdot \overline{\mathbf{r}}+n_{\gamma}\left(\nabla_{\mathbf{u}} B^{\gamma}\right)_{*} \cdot \overline{\mathbf{r}}\right\} \frac{\partial w}{\partial \eta_{\beta}^{\alpha}}\right] \overline{\mathbf{r}}+O\left(\epsilon^{2}\right) \quad \beta=1,2 \ldots, m
\end{aligned}
$$

where

$$
\bar{c}=c_{0}(\mathbf{n}, \mathbf{u}=0)
$$

and we note that $\Psi_{*}^{(\alpha)}$ is zero because $A_{*}$ and $B_{*}^{(\alpha)}$ are constants and

$$
\begin{aligned}
\frac{d w}{d t} & =\bar{\Omega} w+\epsilon\left[\left(\left(\nabla_{\mathbf{u}} \mathbf{l}\right)_{0} \cdot \overline{\mathbf{r}}\right) A_{*} \overline{\mathbf{r}}_{t}+\overline{\mathbf{l}}\left(\left(\nabla_{\mathbf{u}} A\right)_{*} \cdot \overline{\mathbf{r}}\right) \overline{\mathbf{r}}_{t}+\overline{\mathbf{l}} A_{*}\left(\nabla_{\mathbf{u}} \mathbf{r}\right)_{0} \cdot \overline{\mathbf{r}}_{t}\right] w \\
& +\epsilon\left[\left(\left(\nabla_{\mathbf{u}} \mathbf{l}\right)_{0} \cdot \overline{\mathbf{r}}\right) B_{*}^{(\alpha)} \overline{\mathbf{r}}_{x_{\alpha}}+\overline{\mathbf{l}}\left(\left(\nabla_{\mathbf{u}} B^{(\alpha)}\right)_{*} \cdot \overline{\mathbf{r}}\right) \overline{\mathbf{r}}_{x_{\alpha}}+\overline{\mathbf{l}} B_{*}^{(\alpha)}\left(\nabla_{\mathbf{u}} \mathbf{r}\right)_{0} \cdot \overline{\mathbf{r}}_{x_{\alpha}}\right] w \\
& +\epsilon\left\{\overline{\mathbf{l}} A_{*}\left(\left(\nabla_{\mathbf{u}} \mathbf{r}\right)_{0} \cdot \overline{\mathbf{r}}\right) w_{t}+\overline{\mathbf{l}} B_{*}^{(\alpha)}\left(\left(\nabla_{\mathbf{u}} \mathbf{r}\right)_{0} \cdot \overline{\mathbf{r}}\right) w_{x_{\alpha}}\right\} \\
& -\epsilon\left[\left(\Gamma^{t} b_{t}+\Gamma^{\alpha} b_{x_{\alpha}}+\Gamma b\right) w_{\theta}+W b_{t}+V^{\alpha} b_{x_{\alpha}}+D^{\alpha \beta}+E b\right] \\
& +O\left(\epsilon^{2}\right)
\end{aligned}
$$

If the terms of the order $\epsilon$ are also neglected in the ray equations (3.20) and (3.21), these equations decouple from the transport equation (3.22) and give the linear rays. In order to retain the nonlinear effects it is necessary to retain in the ray equations, terms atleast upto order $\epsilon$. The situation for the transport equation (3.22) is different. Exact solution (Ravindran and Prasad, 1985) and numerical results (Prasad and Sangeeta, 1999) show that inclusion of order $\epsilon$ terms in (3.20) and (3.21) changes $\bar{\Omega}$ by order 1 in the caustic region leading to order 1 change in the value of $w$ in finite time. This is in contrast to what we expect in a perturbation method. But it is not surprising when we note that 
the neglect of $O(\epsilon)$ terms (3.20) and (3.21) (i.e. linear theory) changes $\bar{\Omega}$ from a finite curvature to infinite curvature in the caustic region which is reached in finite time. It is different with the transport equation (3.22), which with only the first term on the right hand side always leads to a finite value of $w$ everywhere whenever terms of order $\epsilon$ is included in (3.20-3.21) and $\epsilon \tilde{w}$ not choosen too small. During the competition of convergence of linear rays and opposing effect of nonlinearity, a balance is reached which leads to a finite change in $\bar{\Omega}$. There is no mathematical proof so far for the amplitude to be finite due to nonlinearity but extensive numerical computation with small (but not very small) values of amplitude $\epsilon w$ leads to this conjecture. In all these cases the effect of inclusion of the terms of order $\epsilon$ in (3.22) will remain small in finite time. As stated in the abstract and the end of the introduction, we have indeed deduced a weakly nonlinear theory (i.e. equations (3.20)-(3.21)) in which $w$ has error $O\left(\epsilon^{2}\right)$ (i.e. the solution $\mathbf{u}$ has error $\left.O\left(\epsilon^{3}\right)\right)$. However, in the solution of the simpler WNLRT (i.e. equations (3.20), (3.21) and (3.23)) the amplitude $w$ has error $O(\epsilon)$. Thus, to get only the leading order correction to the amplitude, it is not necessary to retain the last four terms in (3.22) which are multiplied by $\epsilon$ and then we get

$$
\frac{d w}{d t}=\bar{\Omega} w
$$

This transport equation looks exactly the same as the linear transport equation but it contains now all leading order nonlinear effects since in it $\frac{d w}{d t}$ represents time rate of change along the nonlinear rays and $\mathbf{n}$ appearing in $\bar{\Omega}$ is the normal of the nonlinear wavefront. In fact the equation (3.23) along with the equations (3.20) and (3.21) is equivalent to the transport equation

$$
w_{t}+\left\{\overline{\mathbf{l}} B_{*}^{(\alpha)} \overline{\mathbf{r}}+\epsilon\left[\left(\nabla_{\mathbf{u}} \mathbf{l}\right)_{0} \cdot \overline{\mathbf{r}} B_{*}^{(\alpha)} \overline{\mathbf{r}}+\overline{\mathbf{l}}\left(\left(\nabla_{\mathbf{u}} B^{(\alpha)}\right)_{*} \cdot \overline{\mathbf{r}}\right) \overline{\mathbf{r}}+\overline{\mathbf{l}} B_{*}^{(\alpha)}\left(\nabla_{\mathbf{u}} \cdot \mathbf{r}\right)_{0} \overline{\mathbf{r}}\right] w\right\} w_{x_{\alpha}}=\bar{\Omega} w
$$

and $\bar{\Omega}$, which contains derivatives of $\mathbf{n}$ and which is calculated using coupled system (3.20), (3.21) and (3.23) remains finite every where including the points on the caustic, where the corresponding value $\Omega^{*}$ by linear theory tends to infinity. The equations (3.20), (3.21), (3.23) form the final coupled system of equations of our nonlinear ray theory. Retaining the other terms of order $\epsilon$ in (3.22) will modify the results only by effects of order $\epsilon^{2}$ in solution $\mathbf{u}$ since these terms in (3.22) are actually of order $\epsilon^{2}$ in the original equation (2.6). Equations (3.20), (3.21) and (3.23) are exactly the same as the equations obtained for a nonlinear ray theory by Prasad (1994) (see also Prasad (1975), Ravindran and Prasad (1985), Prasad(1993)). In these earlier papers $w$ is of order $\epsilon$, i.e $w$ in those papers is the same as $\epsilon w$ here.

\section{Comparison with other theories}

The WNLRT developed in the last two sections is valid over a length scale $L$ over which the assumptions involved in the derivation of the equations are valid. This length $L$ can be derermined only from the solution of this apporximate theory. One exact solution, called composite simple wave solution in Ravindran and Prasad (1985) and Prasad (1993), and extensive numerical solution by Prasad and Sangeeta (1999) show that this $L$ is large compared to the length scale $R$ of the order of principal radii of curvature of the initial 
wavefront. The Choquet-Bruhat's nonlinear theory is valid over a length scale $L_{c}$ which is small compared to $R$. On this scale $L_{c}$, the linear and nonlinear wavefronts are not only close but have same shape and the amplitude given by the linear theory remains small. Thus $\frac{L_{c}}{R}<<1<<\frac{R}{L}$. We shall show that over the length scale $L_{c}$, the equation (3.23) reduces to the leading order equation obtained from Choquet-Bruhat's theory in addition to some extra terms which can be neglected. We examine the equation (3.23) over a length scale $L_{c}$. On this length scale, the linear wavefront and the corresponding nonlinear wavefront originating from a same initial wavefront are close to one another and their unit normals denoted respectively by $\mathbf{n}_{*}$ and $\mathbf{n}$ differ by a quantity of order $\epsilon$. We denote the rate of change along the linear ray by $\frac{d^{*}}{d s^{*}}$ i.e.

$$
\frac{d^{*}}{d s^{*}}=\mathbf{1}_{*} A_{*}^{\alpha} \mathbf{r}_{*} \frac{\partial}{\partial x^{\alpha}},\left(x_{\alpha}\right)=\left(x_{0}=t, x_{1}=x, \cdots x_{m}=x_{m}\right)
$$

where we have not set $\mathbf{l} A \mathbf{r}=1$, and used $A^{0}=A, A^{\alpha}=B^{(\alpha)}$ and

$$
\mathbf{l}_{*}=\overline{\mathbf{l}}\left(\mathbf{n}_{*}, 0\right), \quad \mathbf{r}_{*}=\overline{\mathbf{r}}\left(\mathbf{n}_{*}, 0\right)
$$

The summation convention in this section extends on the range $0,1,2, \cdots, m$. The rate of change $\frac{d}{d s}$ along the nonlinear ray (see equations (3.23) and (3.24) for $\left|\mathbf{n}-\mathbf{n}_{*}\right|=O(\epsilon)$ can be written as

$$
\begin{aligned}
\frac{d}{d s} & =\mathbf{l}_{*} A_{*}^{\alpha} \mathbf{r}_{*} \frac{\partial}{\partial x_{\alpha}}+\epsilon\left\{\left(\left(\nabla_{\mathbf{n}} \overline{\mathbf{l}}\right)_{*} \cdot\left(\frac{\mathbf{n}-\mathbf{n}_{*}}{\epsilon}\right)\right) A_{*}^{\alpha} \mathbf{r}_{*}+\mathbf{l}_{*} A_{*}^{\alpha}\left(\left(\nabla_{\mathbf{n}} \overline{\mathbf{r}}\right)_{*} \cdot\left(\frac{\mathbf{n}-\mathbf{n}_{*}}{\epsilon}\right)\right)\right\} \frac{\partial}{\partial x_{\alpha}} \\
& +\epsilon w\left[\left(\left(\nabla_{\mathbf{u}} \mathbf{l}\right)_{*} \cdot \mathbf{r}_{*}\right) A_{*}^{\alpha} \mathbf{r}_{*}+\mathbf{l}_{*}\left(\left(\nabla_{\mathbf{u}} A^{\alpha}\right)_{*} \cdot \mathbf{r}_{*}\right) \mathbf{r}_{*}+\mathbf{l}_{*} A_{*}^{\alpha}\left(\left(\nabla_{\mathbf{u}} \mathbf{r}\right)_{*} \cdot \mathbf{r}_{*}\right)\right] \frac{\partial}{\partial x_{\alpha}}+0\left(\epsilon^{2}\right)(4.3)
\end{aligned}
$$

where $\nabla_{\mathbf{n}}=\left(\frac{\partial}{\partial n_{1}}, \ldots, \frac{\partial}{\partial n_{n}}\right)$. The middle term in the square bracket is important and we write it along with the first term on the right hand side of (4.3). Thus

$$
\left.\frac{d}{d s}=\frac{d^{*}}{d s^{*}}+\epsilon\left\{\mathbf{l}_{*}\left(\nabla_{\mathbf{u}} A^{\alpha}\right)_{*} \mathbf{r}_{*}\right) \mathbf{r}_{*}\right\} w \frac{\partial}{\partial x_{\alpha}}+\epsilon w S^{\alpha} \frac{\partial}{\partial x_{\alpha}}+\epsilon T^{\alpha} \frac{\partial}{\partial x_{\alpha}}+0\left(\epsilon^{2}\right)
$$

where

$$
\begin{gathered}
S^{\alpha}=\left(\left(\nabla_{\mathbf{u}} \mathbf{l}\right)_{*} \cdot \mathbf{r}_{*}\right) A_{*}^{\alpha} \mathbf{r}_{*}+\mathbf{l}_{*} A_{*}^{\alpha}\left(\left(\nabla_{\mathbf{u}} \mathbf{r}\right)_{*} \cdot \mathbf{r}_{*}\right) \\
T^{\alpha}=\left(\left(\nabla_{\mathbf{n}} \overline{\mathbf{l}}\right)_{*} \cdot\left(\frac{\mathbf{n}-\mathbf{n}_{*}}{\epsilon}\right)\right) A_{*}^{\alpha} \mathbf{r}_{*}+\mathbf{l}_{*} A_{*}^{\alpha}\left(\left(\nabla_{\mathbf{n}} \overline{\mathbf{r}}\right)_{*} \cdot\left(\frac{\mathbf{n}-\mathbf{n}_{*}}{\epsilon}\right)\right) .
\end{gathered}
$$

The second term in (4.4) contains in it the nonlinear stretching of the rays as given in Choquet-Bruhat's theory. Infact, if we make a transformation from $\left(x_{\alpha}\right)$-coordinates to $\left(\phi^{*}, y^{1}, \ldots, y^{n}\right)$ coordinates (where $\phi^{*}$ is the linear phase function).

$$
\phi^{*}=\phi^{*}\left(x^{0}, x_{1}, \ldots, x_{m}\right), \quad y_{\alpha}=x_{\alpha}, \quad \alpha=1,2, \ldots n
$$

then

$$
\frac{\partial}{\partial x^{0}}=\phi_{x^{0}}^{*} \frac{\partial}{\partial x^{0}}, \frac{\partial}{\partial x_{\alpha}}=\phi_{x_{\alpha}}^{*} \frac{\partial}{\partial \phi^{*}}+\frac{\partial}{\partial y^{\alpha}}, \alpha=1,2, \ldots, n
$$

so that with $\theta^{*}=\frac{\phi^{*}}{\epsilon}$

$$
\epsilon\left\{\mathbf{l}_{*}\left(\left(\nabla_{u} A^{\alpha}\right)_{*} \cdot \mathbf{r}_{*}\right) \mathbf{r}_{*}\right\} w \frac{\partial}{\partial x_{\alpha}}=\mathcal{G} w \frac{\partial}{\partial \theta^{*}}+0(\epsilon)
$$


where

$$
\mathcal{G}=\left\{\mathbf{l}_{*}\left(\phi_{x_{\alpha}}^{*}\left(\nabla_{u} A^{\alpha}\right)_{*} \cdot \mathbf{r}_{*}\right) \mathbf{r}_{*}\right\}
$$

since $\frac{\partial}{\partial \theta^{*}}=\frac{1}{\epsilon} \frac{\partial}{\partial \phi^{*}}$. Further

$$
\epsilon S^{\alpha} \frac{\partial}{\partial x_{\alpha}}=\left\{\left(\left(\nabla_{u} \mathbf{l}\right)_{*} \cdot \mathbf{r}_{*}\right)\left(A_{*}^{\alpha} \phi_{x_{\alpha}}^{*} \mathbf{r}_{*}\right)+\left(\mathbf{l}_{*} A_{*}^{\alpha} \phi_{x_{\alpha}}^{*}\right)\left(\left(\nabla_{u} \mathbf{r}\right)_{*} \mathbf{r}_{*}\right)\right\} \frac{\partial}{\partial \theta^{*}}+0(\epsilon)
$$

in which all terms of order one vanish because $A_{*}^{\alpha} \phi_{x_{\alpha}}^{*} \mathbf{r}_{*}=0$, and $\mathbf{l}_{*} A_{*}^{\alpha} \phi_{x_{\alpha}}^{*}=0$.

On the length scale $L_{c}, \mathbf{n}-\mathbf{n}_{*}=0(\epsilon)$, so that

$$
\epsilon T^{\alpha} \frac{\partial}{\partial x_{\alpha}}=T^{\alpha} \phi_{x_{\alpha}}^{*} \frac{\partial}{\partial \theta^{*}}+0(\epsilon)
$$

and here too all the terms of order one vanish due to the same reason i.e. $A_{*}^{\alpha} \phi_{x_{\alpha}}^{*} \mathbf{r}_{*}=0$ and $\mathbf{l}_{*} A_{*}^{\alpha} \phi_{x_{\alpha}}^{*}=0$. Thus, to the leading order, the transport equation (3.23) or (3.24) reduces to the Choquet-Bruhat's transport equation

$$
\frac{d^{*}}{d t^{*}} w+\mathcal{G} w w_{\theta^{*}}+\Omega_{*} w=0
$$

(See Hunter, 1995). Note that the assumption $\left|\mathbf{n}-\mathbf{n}^{*}\right|=O(\epsilon)$ breaks down as soon the nonlinear wavefront starts approaching a caustic region of the linear theory.

One of the most interesting outcome of this theory is a derivation of the weak shock ray theory Prasad, 1993, (p.95), from the WNLRT consisting of the equations (3.20), (3.21) and (3.24). Shock ray theory consists of the shock ray equations, and an infinite system of compatibility conditions. Unlike the WNLRT, shock ray theory is exact because $\epsilon$ is of the order of the shock thickness which is zero in the inviscid theory and hence the high frequency approximation is exactly satisfied. But the shock ray theory is as difficult as the original problem, infact more difficult due horrendously long expressions present even in the first few (say the second itself) of the infinite number of compatibility conditions involved in it. Infinite number of equations remain involved even if weak shock assumption is made. As mentioned here, the weak shock ray theory can be derived from the WNLRT of this paper. This derivation is not only simple but also much more transparent for the Euler's equations of gasdynamics, which we shall do in the next section. In passing, we mention that an attempt has been made in showing such a relation between WNLRT and shock ray theory by Anile et al (1993) (pp 85-87) without making any distinction between a linear ray, nonlinear ray and shock ray.

\section{Nonlinear waves in a polytropic gas}

Wave propagation in a gas (or in any continuous media) has as its foundation, the three basic conservation laws of physics: conservation of mass, momentum and energy. These laws of physics allow us to derive the field equations which for a polytropic gas are expressed in terms of the fluid velocity $\mathbf{q}$, density $\rho$ and pressure $p$. The equations of motion are

$$
\rho_{t}+\langle\nabla, \rho \mathbf{q}\rangle=0
$$




$$
\mathbf{q}_{t}+\langle\mathbf{q}, \nabla\rangle \mathbf{q}+\frac{1}{\rho} \nabla p=0
$$

and

$$
p_{t}+\langle\mathbf{q}, \nabla\rangle p+\rho a^{2}\langle\nabla, \mathbf{q}\rangle=0
$$

where $a=a(\rho, p)$ is the local speed of sound. The assumption that the gas is polytropic leads to the entropic equation of state

$$
p=K \rho^{\gamma}
$$

in which the coefficient $K$ depends on the entropy and $\gamma$ is the ratio of specific heats, which for air is taken to be 1.4. For such a gas

$$
a^{2}=\frac{\gamma p}{\rho}
$$

These quasilinear equations form a hyperbolic system and are called Euler's equations. For some simplicity in the general theory, we took $\mathbf{u}=0$ to be a basic solution of (2.1) and hence we write the equations of motion by replacing the density $\rho$ by $\rho_{*}+$ $\rho, \quad\left(\rho_{*}=\right.$ constant $)$, pressure $p$ by $p_{*}+p \quad\left(p_{*}=\right.$ constant $)$ and velocity $\mathbf{q}$ by $\mathbf{q}_{*}+\mathbf{q}$, where $\left(\mathbf{q}_{*}=0, \quad \rho=\rho_{*} p=p_{*}\right)$ represents the medium at rest and in uniform state and the symbols $\rho, p$ and $\mathbf{q}$ now represent the perturbations. The equations of motion can be written in the form (2.1) where

$$
\mathbf{u}=\left(\rho, q_{1}, q_{2}, q_{3}, p\right)^{T}, A=I=\text { identity matrix }
$$

and

$$
B_{*}^{(\alpha)}=\left[\begin{array}{ccccc}
q_{\alpha} & \left(\rho_{*}+\rho\right) \delta_{1 \alpha} & \left(\rho_{*}+\rho\right) \delta_{2 \alpha} & \left(\rho_{*}+\rho\right) \delta_{3 \alpha} & 0 \\
0 & q_{\alpha} & 0 & 0 & \frac{1}{\rho_{*}} \delta_{1 \alpha} \\
0 & 0 & q_{\alpha} & 0 & \frac{1}{\rho_{*}} \delta_{2 \alpha} \\
0 & 0 & 0 & q_{\alpha} & \frac{1}{\rho_{*}} \delta_{3 \alpha} \\
0 & \rho_{*} a_{*}^{2}\left(1+\frac{\rho}{\rho_{*}}\right)^{\gamma-1} \delta_{1 \alpha} & \rho_{*} a_{*}^{2}\left(1+\frac{\rho}{\rho_{*}}\right)^{\gamma-1} \delta_{2 \alpha} & \rho_{*} a_{*}^{2}\left(1+\frac{\rho}{\rho_{*}}\right)^{\gamma-1} \delta_{3 \alpha} & q_{\alpha}
\end{array}\right]
$$

where $\delta_{i j}$ is the Kronecker delta, $a_{*}$ is the value of local velocity of sound in the medium at rest. For the wave corresponding to the eigenvalue $\mathbf{q}+\mathbf{n} a$ the eikonal equation is

$$
Q \equiv \phi_{t}+a_{*}\left(1+\frac{\rho}{\rho_{*}}\right)^{\frac{\gamma-1}{2}}|\nabla \phi|+\langle\mathbf{q}, \nabla \phi\rangle=0
$$

and the corresponding right eigen vector is $\mathbf{r}=\left(r_{1}, r_{2}, r_{3}, r_{4}, r_{5}\right)^{T}$ where

$$
r_{1}=\frac{\rho_{*}}{a_{*}}\left(1+\frac{\rho}{\rho_{*}}\right)^{-\frac{\gamma-3}{2}}, r_{2}=n_{1}, \quad r_{3}=n_{2}, \quad r_{4}=n_{3}, \quad r_{5}=\rho_{*} a_{*}\left(1+\frac{\rho}{\rho_{*}}\right)^{\frac{\gamma+1}{2}}
$$


In order that $\mathbf{l} A \mathbf{r}=1$ we choose the left eigen vector as

$$
l_{1}=0, \quad l_{2}=\frac{1}{2} n_{1}, \quad l_{3}=\frac{1}{2} n_{2}, \quad l_{4}=\frac{1}{2} n_{3}, \quad l_{5}=\frac{1}{2 \rho_{*} a_{*}}\left(1+\frac{\rho}{\rho_{*}}\right)^{-\frac{\gamma+1}{2}}
$$

where $\mathbf{n}=\left(n_{1}, n_{2}, n_{3}\right)$ is the unit normal. The ray equations are given by

$$
\frac{d x_{\alpha}}{d t}=q_{\alpha}+n_{\alpha} a_{*}\left(1+\frac{\rho}{\rho_{*}}\right)^{\frac{\gamma-1}{2}}=\chi_{\alpha}, \text { say }
$$

and

$$
\frac{d n_{\alpha}}{d t}=-a_{*} L_{\alpha}\left(1+\frac{\rho}{\rho_{*}}\right)^{\frac{\gamma-1}{2}}-\sum_{\beta=1}^{3} n_{\beta} L_{\alpha} q_{\beta}=\Psi_{\alpha}
$$

where

$$
\mathbf{L} \equiv\left(L_{1}, L_{2}, L_{3}\right)=\nabla-\mathbf{n}\langle\mathbf{n}, \nabla\rangle
$$

The expressions (2.18) and (3.15) to (3.19) when evaluated lead to the following set of equations of WNLRT upto order $\epsilon$

$$
\begin{gathered}
\rho=\epsilon \frac{\rho_{*}}{a_{*}} w, \quad q_{\alpha}=\epsilon n_{\alpha} w, \quad p=\epsilon \rho_{*} a_{*} w \\
\frac{d x_{\alpha}}{d t}=n_{\alpha}\left(a_{*}+\epsilon \frac{\gamma+1}{2} w\right) \\
\frac{d n_{\alpha}}{d t}=-\epsilon \frac{\gamma+1}{2} L_{\alpha} w
\end{gathered}
$$

and

$$
\frac{d w}{d t}=\bar{\Omega} w
$$

where

$$
\bar{\Omega}=-\frac{1}{2} a_{*}\langle\nabla, \mathbf{n}\rangle
$$

is the mean curvature of the nonlinear wavefront and

$$
\frac{d}{d t}=\frac{\partial}{\partial t}+\left(a_{*}+\epsilon \frac{\gamma+1}{2} w\right)<\mathbf{n}, \nabla>
$$

is the time rate of change along the rays given by (5.11) and (5.12). These are the same equations as derived in Prasad (1994) where $w$ is $\epsilon w$ in the above equations. Since $|\mathbf{n}|=1$, only two of the three equations (5.12) are independent. Therefore, the equations (5.11)-(5.13) form a system of 6 coupled equations for the determination of successive positions $\mathbf{x}$ of a nonlinear wavefront, the unit normal $\mathbf{n}$ and the wavefront intensity $w$. In the linear theory, $w$ drops out of the (5.11) and (5.12) so that the ray equations decouple from the amplitude equation (5.13). In this case the rays and the successive positions of the wavefront can be constructed without any reference to the amplitude of the wave. This corresponds to the statement of Huygens' wavefront construction for the propagation of a linear wavefront. In our weakly nonlinear theory, the amplitude is related to the curvature of the wavefront (or the ray tube area) by the equation (5.13). The nonlinear rays stretch due to the presence of $w$ in (5.11) and the wavefront rotates due to a nonuniform distribution of the amplitude on the wavefront (represented by $\mathbf{L} w$ 
in (5.12)). Thus the amplitude of the wave modifies the rays and the wavefront geometry which in turn effects the growth and decay of the amplitude.

Further we note that only the tangential derivatives, on a wavefront $\Omega_{t}$ at a time $t$, of $w$ and $n_{\alpha}$ appear on the right hand side of the equations of WNLRT. Therefore, given the initial position $\Omega_{0}$ of the wavefront and the distribution of the amplitude on it, all quantities on the right hand side of the equations (5.11) - (5.13) can be completely determined at $t=0$ as in the case of a non-characteristic Cauchy problem. Hence, the evolution of the wavefront and the distribution of amplitude on it can be determined from these equations. This implies that, in the short wave approximation, the nonlinear wavefront is self propagating. The result is true not only for a compressible medium but for any continuum medium governed by the hyperbolic system . Huygens' method of wavefront construction has now been very elegantly extended to the construction of a nonlinear wavefront in the short wave limit.

As mentioned at the end of the last section, we shall now derive the shock ray theory for a weak shock from equations (5.11) to (5.15). Consider a weak shock wave propagating into a polytropic gas at rest ahead of it. Assume the shock also to belong to the characteristic field with eigenvalue $\mathbf{q}+\mathbf{n} a$, then shock will be followed by a one parameter family of nonlinear waves governed by the equations (5.11) to (5.13). Each one of these waves will catch up with the shock, interact with it and then disappear. A nonlinear wave while interacting with the shock will be instantaneously coincident with it in the short wave approximation considered in this paper. On the nonlinear wavefront, the transport equation (5.13) remains valid. Now we use the theorem (Prasad, 1993, p 74)

Theorem: For a weak shock, the shock ray velocity components are equal to the mean of the bicharacteristic velocity components just ahead and just behind the shock, provided we take the wavefront generating the characteristic surface to be instantaneously coincident with the shock surface. Similarly, the rate of turning of the shock front is equal to the mean of the rates of turning of such wavefronts just ahead and just behind the shock.

We denote the unit normal to the shock front by $\mathbf{N}$. For the linear wavefront just ahead of the shock and instantaneously coincident with it (this is actually a linear wavefront moving with the ray velocity $\mathbf{N}$ multiplied by the local sound velocity $\left.a_{*}\right) w=0$ and the bicharacteristic velocity is $\mathbf{N} a_{*}$. For the nonlinear wavefront just behind the shock and instantaneously coincident with it, we denote the amplitude $w$ by $\mu$. Then $\mu$, is shock amplitude of the weak shock under consideration. Using the theorem and the results (5.11) and (5.12) with $n=\mathbf{N}$, we get for a point $\mathbf{X}$ on the shock ray

$$
\begin{gathered}
\frac{d \mathbf{x}}{d T}=\frac{1}{2}\left\{a_{*} \mathbf{N}+\mathbf{N}\left(a_{*}+\epsilon \frac{\gamma+1}{2} \mu\right)\right\}=\mathbf{N}\left(a_{*}+\epsilon \frac{\gamma+1}{4} \mu\right) \\
\frac{d \mathbf{N}}{d T}=-\frac{1}{2}\left\{0+\epsilon \frac{\gamma+1}{2} \mathbf{L} \mu\right\}=-\epsilon \frac{\gamma+1}{4} \mathbf{L} \mu
\end{gathered}
$$

where $T$ is the time measured while moving along a shock ray. We take $w=\mu$ and $\mathbf{n}=\mathbf{N}$ in (5.13) and write it as

$$
\begin{aligned}
\frac{d \mu}{d T} \equiv\left\{\frac{\partial}{\partial t}\right. & \left.+\left(a_{*}+\epsilon \frac{\gamma+1}{4} \mu\right)\langle\mathbf{N}, \nabla\rangle\right\} \mu \\
& =-\frac{1}{2} a_{*}\langle\nabla, \mathbf{N}\rangle \mu-\epsilon \frac{\gamma+1}{4} \mu\langle\mathbf{N}, \nabla\rangle w
\end{aligned}
$$


where we note that since $\mu$ is defined only on the shock front (and also on instantaneously coincident nonlinear wavefront behind it but not the other members of the one parameter family of wavefronts following it), the normal derivative $\langle\mathbf{N}, \nabla\rangle \mu$ does not make sense mathematically. We introduce a new variable, defined on the shock

$$
\mu_{1}=\epsilon\langle\mathbf{n}, \nabla\rangle w, \text { on the shock front }
$$

where $\epsilon$ appears to make $\mu_{1}=O(1)$ since we wish to consider variation of $w$ on a length scale over which the fast variable $\theta$ varies.

The equation (5.18) leads to the first compatibility condition along a shock ray

$$
\frac{d \mu}{d T}=\bar{\Omega}_{s} \mu-\frac{\gamma+1}{4} \mu \mu_{1}
$$

where

$$
\bar{\Omega}_{s}=-\frac{1}{2} a_{*}\langle\nabla, \mathbf{N}\rangle
$$

is the value of $\bar{\Omega}$ for the nonlinear wavefront instantaneously coincident with the shock from behind.

To find the second compatibility condition along a shock, we differentiate (5.13) in the direction of $\mathbf{n}$ but on the length scale over which $\theta$ varies. On this length scale, $\mathbf{n}, \bar{\Omega}$ are constants and we get after rearranging some terms,

$$
\begin{gathered}
\left\{\frac{\partial}{\partial t}+\left(a_{*}+\epsilon \frac{\gamma+1}{4} w\right)\langle\mathbf{n}, \nabla\rangle\right\}\langle\mathbf{n}, \nabla\rangle w=-\frac{1}{2} a_{*}\langle\nabla, \mathbf{n}\rangle\langle\mathbf{n}, \nabla\rangle w-\epsilon \frac{\gamma+1}{4} w\langle\mathbf{n}, \nabla\rangle^{2} w \\
-\epsilon \frac{\gamma+1}{4}\{\langle\mathbf{n}, \nabla\rangle w\}^{2}
\end{gathered}
$$

Writing this equation for the wavefront instantaneously coincident with the shock, multiplying it by $\epsilon$ and introducing a variable $\mu_{2}$ by

$$
\mu_{2}=\epsilon^{2}\langle\mathbf{n}, \nabla\rangle^{2} w \quad, \text { on the shock }
$$

we get

$$
\frac{d \mu_{1}}{d T}=\bar{\Omega}_{s} \mu_{1}-\frac{\gamma+1}{4} \mu_{1}^{2}-\frac{\gamma+1}{4} \mu \mu_{2}
$$

which is the second compatibility condition along shock rays given by (5.16) and (5.17).

Similarly, higher order compatibility conditions can be derived. Thus, for the Euler's equations, we have derived the infinite system of compatibility conditions for a weak shock just from the dominant terms of our WNLRT (see pages 85-87, Anile et al, 1993).

As we have already mentioned, the shock ray theory is an exact theory (weak shock assumption is another independent assumption) but it is impossible to use it for computation for shock propagation. Prasad and Ravindran proposed a new theory of shock dynamics (NTSD) in 1990-91 (see Prasad, 1993) according to which the system of equations (5.16), (5.17), (5.20) and (5.23) can be closed by dropping the term containing $\mu_{2}$ from the equations (5.23). The NTSD has been found to be computationally very efficient and gives results which agree well with theoritical results (whatever available), experiment results and results obtained from computation of full gas-dynamics equations (Kevlahan, 1996 and a number of papers from Prasad, Ravindran and their collaborators). This new theory of shock dynamics forms the basis of extensive numerical computation by Monica and Prasad, 2000 to find the nonlinear effects in the linear caustic region.

Acknowledgement: The theory presented in section 2 was developed in collaboration with Prof. J. K. Hunter. The author sincerely thanks him for his valuable contribution. 


\section{References}

[1] M. A. Anile, J. K. Hunter, P. Pantano and G. Russo, Ray Methods for Nonlinear Waves in Fluids and Plasmas, 1993, Longman.

[2] M. Brio and J. K. Hunter, Mach reflection for two-dimensional Burgers equation, Physica D 60 (1992), 194-207.

[3] J. K. Hunter and M. Brio, Weak shock reflection, J.Fluid Mechanics 410 (2000), 235-261.

[4] Y. Choquet-Bruhat, Ondes asymptotique et approachées pour systèmes nonlineares dèquations aux dérivées partielles nonlinéaires, J.Math. Pure et Appl., 48 (1969), 117-158.

[5] J. K. Hunter, Asymptotic equations for nonlinear hyperbolic waves, Surveys in Applied Mathematics, vol II, edited by J.B.Keller, W. McLaughlin and C. Papanicolaou, 1995, Plenum Press.

[6] J. K. Hunter, Nonlinear wave diffraction, Geometrical Optics and Related Topics, edited by Colombini, F. and Lerner, N., 1997, Berkhauser, 221-243.

[7] J. B. Keller, Geometrical acoustics, I : theory of weak shock waves, J. Appl. Phys. 25 (1954), 938-947

[8] N.K.-R. Kevlahan, The propagation of weak shocks in non-uniform flow, J. Fluid Mech 327 (1996), 167-197

[9] Monica, A and Phoolan Prasad, Propagation of a curved weak shock, communicated for publication in J.Fluid Mechanics.

[10] D. F. Parker, Nonlinearity, relaxation and diffusion in acoustic and ultrasonics, J. Fluid Mech. 39 (1969), 793-815

[11] D. F. Parker, An asymptotic theory for oscillatory nonlinear signals, J. Inst. Maths. Applics, 7 (1971), 92-110.

[12] Phoolan Prasad, Approximation of the perturbation equations of a quasilinear hyperbolic system in the neighbourhood of a bicharacteristic, J. of Mathematical Analysis and Applications, 50 (1975), 470-482.

[13] Phoolan Prasad, Extension of Huyghen's construction of a wavefront to a non-linear wave-front and a shock-front, Current Science, 56 (1987), 50-54.

[14] Phoolan Prasad, Propagation of a Curved Shock and Nonlinear Ray Theory, Pitman Researches in Mathematics Series, No. 292 (1993), Longman.

[15] Phoolan Prasad, A nonlinear ray theory, Wave Motion, 20 (1994), 21-31.

[16] Morton, K.W., Prasad, P. and Ravindran, R., Conservation forms of the nonlinear ray equations, Department of Mathematics, Indian Institute of Science, Bangalore, Tech. Rep 2 (1992). 
[17] Phoolan Prasad and Renuka Ravindran, A theory of nonlinear waves in multidimensions with special reference to surface-water-waves, J.Inst. Math. and Applcs., 20 (1977), 9-20.

[18] Phoolan Prasad and K. Sangeeta, Numercial simulation of converging nonlinear wavefronts, J. Fluid Mech. 385 (1999), 1-20.

[19] Renuka Ravindran and Phoolan Prasad, Kinematics of a shock front and resolution of a hyperbolic caustic (a review article), 'ADVANCES IN NONLINEAR WAVES' Vol.II, published by Pitman, London, 1985, 77-99.

[20] B. Sturtevant and V. A. Kulkarny, The focusing of weak shock waves, J. Fluid Mech. 73(1976), 651-671.

[21] E. Tabak and R. R. Rosales, Weak shock focusing and von-Neumann paradox of oblique shock reflection, Phys. Fluids 6 (1994), 1874-1892

[22] G. B. Whitham, On the propagation of weak shock waves, J. Fluid Mech. 1 (1956), 290-318. 